\title{
RESPIRATORY ENZYMES OF THE SILKMOTH ANTHERAEA PERNYI IN RELATION TO METAMORPHOSIS AND PUPAL DIAPAUSE
}

\author{
DAVID G. SHAPPIRIO \\ Department of Zoology, The University of Michigan, Ann Arbor, Michigan 48104, \\ U.S.A.
}

(Received 22 October 1973)

\begin{abstract}
Wing epidermis from diapausing and non-diapausing silkmoths (Antheraea pernyi) was assayed for NADH [nicotinamide adenine dinucleotide (reduced form)] oxidase, NADH- and succinate-cyctochrome c reductases, and cytochrome c oxidase.

2. Diapausing pupae showed a marked decline in activities after pupation and the same subsequent profile of very low activities reported for diapausing Cecropia silkmoths.

3. Non-diapausing pupae showed only a slight and transient decline after pupation and otherwise retained relatively high activities throughout metamorphosis.
\end{abstract}

\section{INTRODUCTION}

THE ABILITY of many organisms to exploit so-called temperate environments depends in no small measure on their capacity for marked seasonal metabolic alterations, which have evolved as major features in the life history. An important illustration is insect diapause (Lees, 1955; Danilevskii, 1965). The present paper extends previous studies on changes in respiratory enzymes in relation to the pupal diapause and metamorphosis of silkmoths. As illustrated by the Cecropia silkmoth, Hyalophora cecropia, the developmental arrest of the diapausing pupa is accompanied by a profoundly attenuated respiratory metabolism (Schneiderman \& Williams, 1953). Cytochromes and other electron transfer components are only barely detectable in most tissues of diapausing $H$. cecropia pupae (Shappirio \& Williams, 1957a, b; Shappirio, 1966). These and other striking metabolic features of the diapausing state, reviewed by Wyatt (1972), arise shortly after the pupa is formed, owing to the endocrine events that establish and enforce the developmental arrest (Williams, 1946, 1952, 1969). When the endocrine system later causes termination of diapause, prominent increases in the respiratory rate and oxidative enzyme activities are early features of the developmental response.

A first objective of the present study is to examine the applicability of previous enzymatic findings, based solely on $H$. cecropia, to other species exhibiting pupal diapause. Of interest are the respiratory enzyme profile during diapause and the transition to the diapausing state. A further objective is to determine whether alterations in respiratory enzymes at the outset of diapause are in fact related to 
arrested development, rather than to other attributes of the pupal stage. This distinction is difficult in $H$. cecropia since diapause is obligatory and embraces essentially the entire pupal period. In the present study, both goals have been sought via enzymatic measurements on the wing epidermis of the Saturniid silkmoth Antheraea pernyi. The wing displays a respiratory enzyme profile typical of the insect's heart, gut and non-muscular tissues, and is also advantageous for biochemical analysis (Shappirio \& Williams, 1957a, b). The suitability of $A$. perny $i$ derives from the ease with which one may obtain comparable diapausing or nondiapausing pupae by manipulation of photoperiod prevailing during larval life (Tanaka, 1944). The photoperiodic control of diapause in $A$. pernyi has subsequently become the subject of intensive investigations by Japanese, Soviet and American workers (see reviews by Lees, 1955; Danilevskii, 1965; Beck, 1968; Williams, 1969). An abstract outlining the behavior of cytochrome $\mathrm{c}$ in metamorphosing $A$. pernyi has also appeared (Shappirio, 1956).

\section{Experimental animals}

\section{MATERIALS AND METHODS}

Diapausing pupae of $A$. pernyi (Guér.) were imported from Japan and maintained under conditions favoring the continuation of diapause (Williams, 1969). Additional A. pernyi, derived from eggs of a British stock originating in eastern Asia, were reared indoors at $18-22^{\circ} \mathrm{C}$ during September and October. Washed oak leaves were supplied and replaced daily. One group of larvae received continuous $24 \mathrm{hr}$ illumination from ordinary incandescent lamps and yielded approximately 98 per cent non-diapausing pupae as evidenced by prompt initiation of adult development within a few days after the pupal ecdysis. Subsequent development was uninterrupted. A second group of larvae, from the same batch of eggs, received continuous illumination during the first two instars, but was then transferred to short-day conditions in which the daily photophase (less than $12 \mathrm{hr}$ ) was derived by natural illumination from the outdoors. Approximately 80 per cent of the resulting pupae entered pupal diapause as shown by their failure to initiate adult development for at least 2 months following the pupal ecdysis. In all cases, the initiation of adult development was verified by microscopic examination to detect the first externally visible retraction of facial and leg epidermis from overlying pupal cuticle. In studies on diapausing pupae, animals were rejected if they showed an oxygen consumption greater than $15 \mu 1 / \mathrm{hr}$ per $\mathrm{g}$ live weight, which might have indicated the incipient termination of diapause. In studies on animals at the outset of diapause, prospectively non-diapausing pupae were distinguished and rejected if they showed a reduced adherence of epidermis to pupal cuticle or a "mushy" texture to the fat body; both characteristics signal incipient morphogenetic changes associated with adult development (Krishnakumaran et al., 1967).

Some prospectively non-diapausing pupae were caused to enter diapause by removal of their brains within a day after pupal ecdysis. The procedure followed that of Williams (1946, 1959) with special attention to rapid surgery and prompt implantation of phenylthiourea to counteract the potent phenoloxidase activity in the hemolymph of these insects. Enzymatic measurements on brainless pupae were deferred at least 1 month after surgery, to afford time for any injury-induced elevation in respiratory enzymes (Shappirio, 1960; Shappirio \& Harvey, 1965), to subside to the normal diapausing level.

\section{Preparative procedures and enzyme assays}

All preparative procedures were carried out on ice or under refrigeration to maintain a temperature close to $0^{\circ} \mathrm{C}$. Wings were isolated by dissection in $320 \mathrm{mM}$ sucrose containing a few crystals of phenylthiourea. Excess hemolymph was removed by gentle rinsing in fresh 
sucrose. Wings from six to eight diapausing pupae were pooled to provide adequate material; two to three individuals sufficed in the case of non-diapausing animals. After homogenization in $320 \mathrm{mM}$ sucrose (A. H. Thomas No. 3431-A homogenizer, clearance $0.006 \mathrm{in} ., 600 \mathrm{rev} / \mathrm{min}$, twenty strokes) a particulate fraction including mitochondria and substantial microsomal material was isolated as described by Shappirio \& Williams (1957b). Sorvall RC-2 and M.S.E. refrigerated centrifuges were utilized. Efforts to prepare cleaner mitochondrial fractions were abandoned due to excessive loss of material. Final particulates were suspended in $0.1 \mathrm{M}$ phosphate $\left(\mathrm{pH} \mathrm{7.2)}\right.$ ) and assayed at $25^{\circ} \mathrm{C}$ for the following systems, using spectrophotometric procedures described by Shappirio \& Williams (1957b): NADH [nicotinamide adenine dinucleotide (reduced form)] oxidase, NADH- and succinatecytochrome c reductases, and cytochrome c oxidase. Hilger "Uvispek", Beckman DU and Aminco-Chance spectrophotometers were used. Under these conditions, reaction rates were dependent upon enzyme concentration and were linear for at least 3-5 min. Activities were expressed on a nitrogen basis using the Folin-Ciocalteau phenol reagent in the micromodification of Kabat \& Mayer (1948); this spectrophotometric method was calibrated via parallel micro-Kjeldahl analyses on representative samples of particulate fractions isolated from diapausing and non-diapausing $A$. pernyi.

\section{RESULTS}

\section{Respiratory enzymes in diapausing pupae}

Table 1 records the levels of the four oxidative enzyme systems examined in this study, during the time span from just less than 2 days after the pupal ecdysis to several months thereafter. In prospectively diapausing pupae of the British stock, previously reared under short-day conditions, the activities recorded at $42-48 \mathrm{hr}$

TABle 1-RespiRatoRY ENZYME SYSTEMS IN WINGS OF DIAPAUSING PUPAE

\begin{tabular}{|c|c|c|c|c|c|c|}
\hline \multirow[b]{2}{*}{ Animals } & \multirow[b]{2}{*}{ Assay time } & \multicolumn{4}{|c|}{ Enzymatic activity } & \multirow[b]{2}{*}{ No. } \\
\hline & & NO & SCR & NCR & $\mathrm{CO}$ & \\
\hline $\begin{array}{l}\text { "British" stock } \\
\text { reared under } \\
\text { short daylength }\end{array}$ & $\begin{array}{l}42-48 \mathrm{hr} \\
1 \text { week } \\
6-8 \text { weeks }\end{array}$ & $\begin{array}{c}13 \pm 2 \\
<2 \cdot 5 \pm 0 \cdot 5 \\
<2 \cdot 0 \pm 0 \cdot 5\end{array}$ & $\begin{array}{c}19 \pm 3 \\
5 \cdot 2 \pm 1 \cdot 0 \\
7 \cdot 4 \pm 2 \cdot 6\end{array}$ & $\begin{array}{l}95 \pm 15 \\
36 \pm 4 \\
16 \pm 3\end{array}$ & $\begin{array}{c}330 \pm 30 \\
125 \pm 15 \\
71 \pm 9\end{array}$ & $\begin{array}{l}3 \\
3 \\
3\end{array}$ \\
\hline Japanese stock & $\begin{array}{l}\text { 8-12 } \\
\quad \text { weeks }\end{array}$ & $<3 \cdot 0 \pm 0.5$ & $7 \cdot 9 \pm 2 \cdot 6$ & $38 \pm 11$ & $110 \pm 16$ & 3 \\
\hline $\begin{array}{l}\text { "British" stock reared } \\
\text { under long daylength; } \\
\text { brainless }\end{array}$ & $\begin{array}{l}\text { 4-20 } \\
\text { weeks }\end{array}$ & $<3.0 \pm 0.5$ & $9 \cdot 2 \pm 0 \cdot 8$ & $36 \pm 1$ & $260 \pm 55$ & 3 \\
\hline
\end{tabular}

Abbreviations and units of activity for each enzyme system: NO $=$ NADH oxidase (units $=$ nmoles $\mathrm{NADH}$ oxidized/min per mg nitrogen); $\mathrm{SCR}, \mathrm{NCR}=$ succinate-, NADH-cytochrome $\mathrm{c}$ reductases (units $=$ nmoles cytochrome $\mathrm{c}$ reduced/min per $\mathrm{mg}$ nitrogen); $\mathrm{CO}=$ cytochrome $\mathrm{c}$ oxidase (units $=$ nmoles cytochrome $\mathrm{c}$ oxidized/min per $\mathrm{mg}$ nitrogen). All activities expressed as means \pm average deviation. Assay time: after termination of pupal ecdysis. Number of replicate experiments shown in right-hand column. When NADH oxidase activity was below a detectable level, the table records "less than" the activity which would have been minimally detectable under the experimental conditions. 
were relatively high and almost equivalent to those of prospectively non-diapausing pupae just after the pupal ecdysis. But, as shown in Table 1, the insects destined for diapause showed substantially lower levels of activity 1 week after pupation. This was particularly evident for NADH oxidase, which was below a detectable level; but a marked decline also occurred in the case of the other three enzyme systems, their activity falling to a level roughly one-third of that encountered shortly after pupation. Table 1 demonstrates that these lower levels persisted with only slight further change during the ensuing 2 months of diapause. It is further evident that activities in diapausing pupae of the Japanese stock were not readily distinguishable from those already described. The final group of animals considered in Table 1 are the presumptively non-diapausing pupae, previously reared under long day conditions, in which diapause was surgically enforced; here, Table 1 reveals that the levels of activity were low and closely similar to those recorded earlier for diapausing pupae, except for cytochrome $c$ oxidase which was somewhat elevated.

\section{Respiratory enzymes in non-diapausing individuals}

Shown in Table 2 is the behavior of the four oxidative enzyme systems in non-diapausing $A$. pernyi. Here, the brief pupal period leads within several days to the initiation of adult development and the pharate adult phase that encompasses final morphogenesis of the adult moth. During the first day after pupal ecdysis, and thereafter, each enzyme system displays a substantially greater activity than described earlier for diapausing pupae. Table 2 suggests a decline in activity of all systems other than cytochrome c oxidase, starting midway during the second day after pupal ecdysis and embracing approximately the 37-50 hr period. The observation that three of the four systems declined, while the fourth scarcely if at all, suggests that any change is attributable to enzymes and not to the nitrogen baseline; fluctuation in the latter would presumably reflect itself in all four enzyme systems. Despite this apparent decrease, the levels never declined to those characteristic of diapausing pupae 1 week or more after the pupal ecdysis. Subsequent to the second day of pupal life, Table 2 shows that all enzyme systems regained or maintained activities closely similar to those observed just after pupation. Within the rather substantial limits of experimental variation from one preparation to the next, these levels persisted without noteworthy change up to the point late in adult development at which measurements were concluded.

\section{DISCUSSION}

\section{Respiratory enzyme profile during pupal diapause}

It has long been known that the pupal diapause of lepidopterous insects involves a markedly reduced respiratory metabolism (e.g. Heller, 1926). Somewhat more recently, studies on $H$. cecropia have established that the decline in respiratory rate at the outset of diapause is accompanied by a prominent decline in spectroscopically 


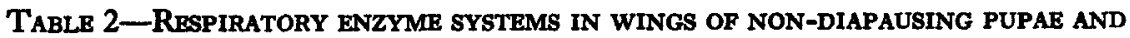
DEVELOPING ADULTS

\begin{tabular}{lrrrr}
\hline & \multicolumn{4}{c}{ Enzymatic activity } \\
\cline { 2 - 5 } $\begin{array}{c}\text { Animals } \\
\text { (time after pupal ecdysis) }\end{array}$ & NO & SCR & NCR & CO \\
\hline (hr) & 11 & 17 & 110 & 390 \\
0 & 27 & 38 & 180 & 530 \\
$1-2$ & 26 & 32 & - & 410 \\
$8-11$ & 20 & 16 & 140 & 300 \\
$13-14$ & 27 & 39 & 180 & 580 \\
$21-25$ & 11 & 21 & - & 270 \\
25 & 5 & 9 & 37 & 360 \\
$37-41$ & 5 & 19 & 79 & 290 \\
$48-52$ & 3 & 15 & 68 & 290 \\
$49-50$ & 6 & 23 & 130 & 350 \\
$66-69$ & 8 & 22 & 100 & 470 \\
$67-69$ & & & & \\
& & & & \\
(days) & 11 & 18 & 95 & 1100 \\
5 & 11 & 32 & 140 & 470 \\
5 & 11 & 42 & 220 & 740 \\
7 & 8 & 33 & 220 & 530 \\
7 & 12 & 47 & 120 & 680 \\
10 & 16 & 35 & 170 & 480 \\
14 & & & & \\
\hline
\end{tabular}

Abbreviations and units of activity for each enzyme system as in Table 1. Shown above are results of individual determinations at successive times after the pupal ecdysis. Blank spaces for NCR at 8-11 hr and $25 \mathrm{hr}$ signify absence of these particular measurements.

detectable levels of cytochromes $a+a_{3}, b, c$ and $b_{5}$ in tissues other than intersegmental muscles, and by corresponding decreases in activity of all four oxidative enzyme systems presently studied (Shappirio \& Williams, 1957a, b). Each enzyme system depends on electron transport via one or more of the aforementioned hemoproteins. In the absence of injury (Shappirio \& Harvey, 1965) the low levels of respiratory catalysts persist until diapause is terminated. The first objective of the present study was to examine the generality of previous findings on respiratory enzymes in $H$. cecropia. On the basis of the observations herein reported, together with comparable findings on the related Saturniid silkmoth Samia cynthia (Shappirio, 1974), one can conclude that this respiratory enzyme profile is probably a general characteristic in most individual tissues of diapausing lepidopterous pupae. Thus, an important biochemical adjustment to the diapausing state appears to involve declines in level and activity of respiratory catalysts, rather than only a slowing-down of respiration via short-term respiratory control mechanisms.

In the study of Shappirio \& Williams (1957b), NADH oxidase and succinatecytochrome $c$ reductase activities were both below detectable levels during pupal 
diapause. Much uncertainty surrounded the estimation of NADH oxidase activity due to the inherently low activity coupled with spectrophotometric instability and optical changes in the enzyme preparation. With the greater stability, sensitivity and precision of the Hilger and Aminco-Chance instruments used in the present study on the Japanese and British stocks of $A$. pernyi, NADH oxidase remained undetectable. It is possible that reduced accessibility to substrate during diapause, or losses in cytochrome c during isolation, still yield problems in measurement of NADH oxidase. In $H$. cecropia, NADH oxidase activity becomes just marginally detectable by present techniques. By contrast with the 1957 study, succinatecytochrome $\mathrm{c}$ reductase activity was readily detectable though low in titer, both in the present study on $A$. pernyi and that of Shappirio \& Harvey (1965) on $H$. cecropia. This discrepancy is probably attributable to inactivation of succinate dehydrogenase during the 1957 study, in which conditions did not permit rigorous maintenance of low temperatures. This has subsequently been found to lead to reduced or absent activity, although succinate-cytochrome $\mathrm{c}$ reductase may be reactivated upon exposure to the succinate substrate for $10-15 \mathrm{~min}$ at $25^{\circ} \mathrm{C}$. An apparently similar observation was noted by Singer \& Kearney (1957) with vertebrate succinate dehydrogenase.

\section{Transition to and from the diapausing state}

Although a marked decrease in enzymatic activities of preparations from $A$. pernyi occurred within a week after pupal ecdysis, present data contrast with those obtained in the case of $H$. cecropia, wherein the changes were sooner and more precipitous (Shappirio \& Williams, 1957b). The difference evidently does not reside in the inclusion of non-diapausing animals in assays on pupae supposedly destined for diapause. Data on $A$. pernyi are in conformity with respirometric measurements at the outset of pupal life; these measurements show a steady decline in respiratory rate toward low levels, which were approached 1-2 weeks after pupation. A similar, gradual respiratory decline occurs in $H$. cecropia (Schneiderman \& Williams, 1953) and also characterizes the decrease in various biosynthetic activities to their residual levels during diapause (Krishnakumaran $e t$ al., 1967).

Present data afford insight only into changes in enzymatic activity and do not elucidate the underlying mechanisms. Previous studies, especially those providing spectroscopic evidence for alterations in cytochrome levels at the onset and termination of diapause, have led to widespread usage of the terms "breakdown" and "resynthesis". Though this clearly represents one attractive hypothesis, it is the philosophy of this laboratory that careless application of Ockham's razor may shave off the truth. In fact, breakdown and resynthesis have never been rigorously demonstrated. For example, we do not know if the apoproteins of individual cytochromes are disassembled and resynthesized, de novo, at the outset and termination of diapause. The only study on post-diapausing silkmoths which demonstrates de novo synthesis of the apoprotein of cytochrome $\mathrm{c}$ (Chan \& Margoliash, 1966) actually provides evidence for synthesis associated with the 
fabrication of the elaborate thoracic flight musculature of the adult moth. This synthesis occurs relatively late in adult development and would intuitively be expected because the musculature only exists during pupal life in the form of undifferentiated myoblast precursors. The molecular biology surrounding the mitochondrial components at the onset and termination of diapause remains essentially a mystery, particularly in view of electron microscopic observations that mitochondria show but modest changes (Willis, 1966) and that the ultrastructure and abundance of mitochondrial membrane subunits as visualized by negative staining, remain apparently unaltered during times of change in respiratory enzyme activity (J. Michejda, D. S. Smith \& D. G. Shappirio, unpublished observations).

\section{Contrast between diapausing and non-diapausing insects}

The second major objective of the present study was to determine whether the respiratory transition at the outset of pupal life in $H$. cecropia was indeed related to the onset of diapause, as opposed to one of numerous physiological or biochemical changes related to the onset of pupal life per se, such as those reported elsewhere for other tissues in the non-diapausing skipper, Calpodes ethlius (Locke \& Collins, 1965,1968 ; Larsen, 1970). 'The present objective has largely been met via studies on non-diapausing $A$.pernyi. It is clear that in wings of non-diapausing pupae the levels of oxidative enzyme activity do not fall to the very low levels which are now seen to be generally characteristic during pupal diapause. Thus the alterations previously reported for $H$. cecropia may be ascribed to the onset and termination of diapause. This conclusion conforms exactly with that of a parallel study in which a non-diapausing genetic strain of $S$. cynthia exhibited high and essentially unchanging levels of oxidative enzymes from the time of pupal ecdysis until shortly before emergence of the adult moth (Shappirio, 1974).

To use the felicitous term of the late Ernst Scharrer, it is the animal's "photoneuro-endocrine system" which mediates between the environmental signal and the developmental result. Whether or not diapause occurs in $A$. pernyi depends on the integration and storage of photoperiodic and other environmental information by the larval brain (Williams, 1969). In the present study, the fact that 80 per cent of the pupae underwent diapause, despite exposure to long-day conditions in the first two larval instars, confirms Tanaka's finding that these instars are not of overriding importance for induction of diapause (see discussions by Lees, 1955; Danilevskii, 1965). In insects destined for diapause, the brain of the freshly pupated animal fails to produce the hormone that drives the prothoracic glands, which then become inactive causing arrested development among individual tissues (Williams, 1952). In non-diapausing pupae the brain or prothoracic glands remain active or renew activity after a brief pause (Williams, 1952). Possibly the transient dip seen in enzymatic activities of non-diapausing $A$. pernyi on the second day of pupal life results from such an endocrine pause. But the overall hormonal milieu of non-diapausing pupae directly or indirectly supports the host of biosynthetic and other activities requisite for continued development, among which is a maintenance of underlying aerobic energy-transforming systems. By 
contrast, withdrawal of endocrine support leads to the endergonic and exergonic quiescence of diapause. The respiratory events are part of a complex web of intracellular regulatory mechanisms dependent on the interaction of prothoracic gland hormone with responding cells. In nature, these mechanisms permit successive seasonal generations of $A$. pernyi and similar insects to exploit the alternatives of continuous pupal development in spring or early summer, and arrested development later on.

Acknowledgements-For advice and critical discussions at various phases in this study I wish to thank S. K. Chan, E. F. Hartree, D. Keilin, A. D. Lees, R. Weinberg and C. M. Williams. L. H. Finlayson generously donated silkmoth eggs. E. B. Shappirio collaborated in rearing silkmoths, and together with C. M. Williams carried out some of the surgery. D. C. Ferguson and R. W. Hodges kindly provided access to the U.S. national collections for purposes of insect identification. The work was initiated at the University of Cambridge under NSF support; its continuation at The University of Michigan was supported, in part, by NIH and by funds from the University's Institute of Science and Technology. Importation of $A$. perny $i$ was authorized by the U.S. and Michigan Departments of Agriculture.

\section{REFERENCES}

Beck S. D. (1968) Insect Photoperiodism. Academic Press, New York.

Chan S. K. \& MARGoliash E. (1966) Biosynthesis of cytochrome $\mathrm{c}$ in developing pupae of Samia cynthia. F. biol. Chem. 241, 2252-2255.

Danilevskil A. S. (1965) Photoperiodism and Seasonal Development of Insects. Oliver \& Boyd, Edinburgh.

Heller J. (1926) Chemische Untersuchungen über die Metamorphose der Insekten-III. Mitteilung: über die "subitane" und "latente" Entwicklung. Biochem. Z. 169, 208-234.

Kabat E. A. \& MAYER M. M. (1948) Experimental Immunochemistry, pp. 322-323. Charles C. Thomas, Springfield, Ill.

Krishnakumaran A., Berry S. J., Oberlander H. \& Schneiderman H. A. (1967) Nucleic acid synthesis during insect development--II. Control of DNA synthesis in the Cecropia silkworm and other Saturniid moths. F. Insect Physiol. 13, 1-57.

LARSEN W. J. (1970) Genesis of mitochondria in insect fat body. F. cell. Biol. 47, 373-383.

Lees A. D. (1955) The Physiology of Diapause in Arthropods. Cambridge University Press.

Locke M. \& Collins J. V. (1965) The structure and function of protein granules in the fat body of an insect. F. cell Biol. 26, 857-884.

Locke M. \& Collins J. V. (1968) Protein uptake into multivesicular bodies and storage granules in the fat body of an insect. F. cell Biol. 36, 453-483.

Schneiderman H. A. \& Williams C. M. (1953) The physiology of insect diapause-VII. The respiratory metabolism of the Cecropia silkworm during diapause and development. Biol. Bull. mar. biol. Lab., Woods Hole 105, 320-334.

Shappirio D. G. (1956) Hormonal control of cytochrome c synthesis in silkworms. Résumés, $X X$ th Physiological Congress, Brussels, p. 823.

Shappirio D. G. (1960) Oxidative enzymes and the injury metabolism of diapausing Cecropia silkworms. Ann. N.Y. Acad. Sci. 89, 537-548.

Shappirio D. G. (1966) Pyridine nucleotide levels in silkmoth fat body during the onset, maintenance, and termination of diapause. F. cell Biol. 31, 104A.

Shappirio D. G. (1974) Comparative studies of oxidative enzyme systems in epidermis and fat body of diapausing and non-diapausing silkmoths. F. Insect Physiol. 20, 291-300.

Shappirio D. G. \& HaRveY W. R. (1965) The injury metabolism of the Cecropia silkwormII. Injury-induced alterations in oxidative enzyme systems and respiratory metabolism of the pupal wing epidermis. F. Insect Physiol. 11, 305-327. 
Shappirio D. G. \& Williams C. M. (1957a) The cytochrome system of the Cecropia silkworm-I. Spectroscopic studies of individual tissues. Proc. R. Soc. B 147, 218-232.

Shappirio D. G. \& Williams C. M. (1957b) The cytochrome system of the Cecropia silkworm-II. Spectrophotometric studies of oxidative enzyme systems in the wing epithelium. Proc. R. Soc. B 147, 233-246.

SiNGER T. P. \& KEARNEY E. B. (1957) Determination of succinic dehydrogenase activity. In Methods of Biochemical Analysis (Edited by Glick D.), Vol. 4, pp. 307-333. Interscience, New York.

TaNAKA Y. (1944) Effect of daylength on hibernation of the Chinese oak silkworms. Agriculture Hort., Tokyo (Nogyo oyobi Engei) 19, No. 9. (Cited by LEEs, 1955.)

Williams C. M. (1946) Physiology of insect diapause: the role of the brain in the production and termination of pupal dormancy in the giant silkworm Platysamia cecropia. Biol. Bull. mar. biol. Lab., Woods Hole 90, 234-243.

Williams C. M. (1952) Physiology of insect diapause-IV. The brain and prothoracic glands as an endocrine system in the Cecropia silkworm. Biol. Bull. mar. biol. Lab., Woods Hole 103, 120-138.

Williams C. M. (1959) The juvenile hormone-I. Endocrine activity of corpora allata of the adult Cecropia silkworm. Biol. Bull. mar. biol. Lab., Woods Hole 116, 323-338.

Williams C. M. (1969) Photoperiodism and the endocrine aspects of insect diapause. Symp. Soc. exp. Biol. 23, 285-300. Cambridge University Press.

WiLLIS J. H. (1966) The micromorphology of the Cecropia wing following pupation, during diapause, following injury, and at the onset of adult development. $\mathcal{F}$. Insect Physiol. 12, 933-948.

Wyatт G. R. (1972) Insect hormones. In Biochemical Actions of Hormones (Edited by Litwack G.), Vol. 2, pp. 385-490. Academic Press, New York.

Key Word Index-Dormancy; diapause; development; metamorphosis; hormonal control; endocrine control; photoperiodic control; insect hormones; respiratory enzymes; oxidative enzymes; electron transport; cytochromes; metabolism; silkmoths; Lepidoptera; insects; Arthropods; invertebrates; physiological ecology; biochemical ecology. 